\title{
Stratifications, Equisingularity and Triangulation
}

\author{
David Trotman
}

\begin{abstract}
This text is based on 3 lectures given in Cuernavaca in June 2018 about stratifications of real and complex analytic varieties and subanalytic and definable sets. The first lecture contained an introduction to Whitney stratifications, KuoVerdier stratifications and Mostowski's Lipschitz stratifications. The second lecture concerned equisingularity along strata of a regular stratification for the different regularity conditions: Whitney, Kuo-Verdier, and Lipschitz, including thus the ThomMather first isotopy theorem and its variants. (Equisingularity means continuity along each stratum of the local geometry at the points of the closures of the adjacent strata.) A short discussion follows of equisingularity for complex analytic sets including Zariski's problem about topological invariance of the multiplicity of complex hypersurfaces and its bilipschitz counterparts. In the real subanalytic (or definable) case we mention that equimultiplicity along a stratum translates as continuity of the density at points on the stratum, and quote the relevant results of Comte and Valette generalising Hironaka's 1969 theorem that complex analytic Whitney stratifications are equimultiple along strata. The third lecture provided further evidence of the tameness of Whitney stratified sets and of Thom maps, by describing triangulation theorems in the different categories, and including definable and Lipschitz versions. While on the subject of Thom maps we indicate examples of their use in complex equisingularity theory and in the definition of Bekka's (c)-regularity. Some very new results are described as well as old ones.
\end{abstract}

\section{Stratifications}

Consider some singular spaces which are real algebraic varieties.

David Trotman

Aix-Marseille University, CMI, 39 rue Joliot-Curie, 13453 Marseille Cedex 13, France, e-mail:

david.trotman@univ-amu.fr 
(i) Let $V$ be the curve $\left\{y^{2}=x^{2}+x^{3}\right\}$. Then $V$ has a double point singularity at the origin in $\mathbb{R}^{2}$ (Figure 1 )

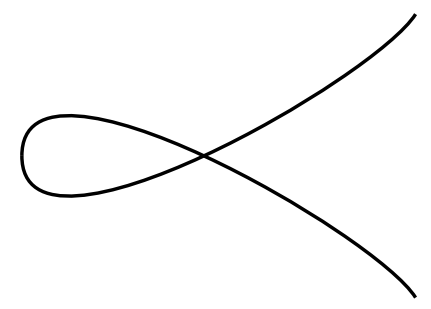

Fig. 1: $y^{2}=x^{2}+x^{3}=0$

(ii) Let $V$ be the curve $\left\{y^{2}=x^{3}\right\}$. Here $V$ has a cusp singularity at the origin in $\mathbb{R}^{2}$.

(iii) Let $V$ be the surface $\left\{z^{2}=x^{2}+y^{2}\right\}$ in $\mathbb{R}^{3}$. This is a cone with an isolated singularity at the origin.

(iv) Let $V$ be the variety $\left\{z\left(x^{2}+(y+z)^{2}\right)=0\right\}$ in $\mathbb{R}^{3}$. This is the union of a plane $P$ and a transverse line $\ell$ (Figure 2).

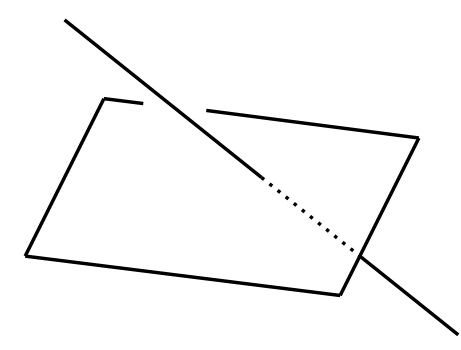

Fig. 2: $z\left(x^{2}+(y+z)^{2}\right)=0$

In each of these four examples the singular set of the variety $V$ is a point. However in Example (iv) the regular part of $V$ is not equidimensional - both 1 and 2 occur as local dimensions. In the other examples the regular part is equidimensional.

Now we give an example of a surface whose singular set is a line.

(v) Let $V$ be $\left\{y^{2}=t^{2} x^{2}-x^{3}\right\}$ in $\mathbb{R}^{3}$. Then the singular set of $V$ is the line $\langle O t>$ (Figure 3)

\subsection{Whitney's conditions (a) and (b)}

We will "stratify" our singular spaces $X$ (closed subsets of some $\mathbb{R}^{n}$ ) by expressing them as a union of smooth manifolds defined by means of a filtration by closed 


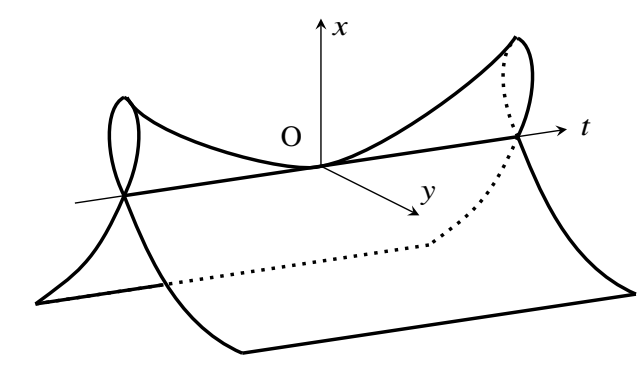

Fig. 3: $y^{2}=t^{2} x^{2}-x^{3}=0$

subsets :

$$
X=X^{d} \supseteq X^{d-1} \supseteq \cdots \supseteq X^{1} \supseteq X^{0} \supseteq X^{-1}=\emptyset
$$

where each difference $X^{j}-X^{j-1}$ is either a smooth manifold of dimension $j$, or is empty. Each connected component of $X^{j}-X^{j-1}$ is called a stratum of dimension $j$.

In Example (iv) the natural filtration can be either

$$
V \supset \ell \supset\{0\} \supset \emptyset
$$

or

$$
V \supset \ell \supset \emptyset=\emptyset \text {. }
$$

Because the intersection point 0 is different from other points on the line $\ell$ we like to take the first filtration. The natural 1-dimensional stratum is thus $\ell \backslash\{0\}$. Also, in Example (v) the natural 1-dimensional strata are the two components of $<O t>\backslash\{0\}$, because 0 is a different point. The local topology of $V$ at points of the $t$-axis changes as we pass through $t=0$.

Question. How can we formalise this difference ?

Whitney ( $\$ 19$ in [Whi65a]; $\$ 8$ in [Whi65b]) defined two regularity conditions (a) and $(b)$.

Let $X, Y$ be two strata (disjoint smooth sub manifolds of $\mathbb{R}^{n}$ ) and let $y_{0} \in Y \cap \bar{X} \backslash X$. Then condition $(a)$ holds for $(X, Y)$ at $y_{0}$ if given any sequence of points $x_{i} \in$ $X$ tending to $y_{0}$, such that the tangent spaces $T_{x_{i}} X$ tend to $\tau$ in the appropriate grassmannian, then $T_{0} Y \subseteq \tau$

If we stratify Example (iv) without removing the point 0 from the line $\ell$, then Whitney's condition $(a)$ fails to hold for the pair of strata $(P-\{0\}, \ell)$ at $0 \in \ell$, where $P$ is the plane $\{z=0\}$.

Look now at Example (v) (the Whitney cusp). We can stratify $V$ by the filtration

$$
V \supset<O t>\supset \emptyset
$$

and then Whitney's condition $(a)$ holds for $(V \backslash<O t>,<O t>)$ at all points. So we need to impose more regularity so that the point $\{0\}$ becomes a stratum : the local topology of slices $\{t=$ constant $\} \cap V$ changes at $t=0$. 
We say that condition $(b)$ holds for $(X, Y)$ at $y_{0} \in Y \cap(\bar{X}-X)$ if given sequences $x_{i} \in X$ and $y_{i} \in Y$ both tending to $y_{0}$, such that $T_{x_{i}} X$ tends to $\tau$ and $y_{i} x_{i} /\left\|y_{i} x_{i}\right\|$ tends to $\lambda$, then $\lambda \in \tau$.

Look at Example (v). A sequence on $V \cap\{y=0\}=\left\{x\left(t^{2}+x\right)=0\right\}$, i.e. $x=-t^{2}$, has $\lambda=(1: 0: 0)$ and $\tau=(1,0,0)^{\perp}$ (the $(t, y)$-plane), so that $\lambda \notin \tau$, and condition (b) fails to hold.

Definition 1.1 A locally finite stratification of a closed set $Z \subseteq \mathbb{R}^{n}$ is called a Whitney stratification if every adjacent pair of strata satisfy condition (b) of Whitney.

Lemma 1.2 Condition (b) implies condition (a).

The proof is an exercise.

Theorem 1.3 (Theorem 2.B.1 in [Tho69], Corollary 10.5 in [Mat12]) A Whitney stratification automatically satisfies the frontier condition, i.e., whenever a stratum $Y$ intersects the closure of a stratum $X$, then $Y$ is contained in the closure of $X$.

Remark 1.4 In Example (iv) the stratification

$$
V \supset \ell \supset \emptyset
$$

does not satisfy the frontier condition. In Example (v), stratifying by

$$
V \supset<O t>
$$

there are 4 strata of dimension 2 (recall that the strata are the connected components of $\left.V^{2} \backslash V^{1}\right)$

Let $X_{1}=\{V \cap\{x \leq 0\} \cap\{y \leq 0\}\}, X_{2}=\{V \cap\{x \leq 0\} \cap\{y \geq 0\}\}, X_{3}=$ $\{V \cap\{t \leq 0\} \cap\{x \geq 0\}\}$ and $X_{4}=\{V \cap\{t \geq 0\} \cap\{x \geq 0\}\}$. These are the 4 strata of our stratification (Figure 4) We see that $Y \cap X_{3} \neq \emptyset$, but that $Y$ is not a subset of $\overline{X_{3}}$, and similarly for $X_{4}$, so that the frontier condition fails for $\left(X_{3}, Y\right)$ and for $\left(X_{4}, Y\right)$. However the frontier condition holds for the pairs of adjacent strata $\left(X_{1}, Y\right)$ and $\left(X_{2}, Y\right)$.

Theorem 1.5 ([Whi65a], Theorem 19.2) Every analytic variety $V$ (real or complex) admits a Whitney stratification.

In fact this is also true for more general sets : for semialgebraic sets (Lojasiewicz [Loj65], Thom [Tho65], Wall [Wal75], Kaloshin [Kal05]), more generally for subanalytic sets (Hironaka [Hir73], Hardt [Har75], Verdier [Ver76], Denkowska, Wachta and Stasica [DWS85]), and even more generally for definable sets in o-minimal structures (Loi [Loi98], van den Dries and Miller [vdDM96], Nguyen, Trivedi and Trotman [NTT14], and Halupczok [Hal14a], [Hal14b]).

One says a regularity condition is generic if every variety (or semialgebraic set, etc.) admits a stratification such that every pair of adjacent strata satisfy the regularity condition.

So Whitney's condition ( $a$ ) and Whitney's condition $(b)$ are generic. 


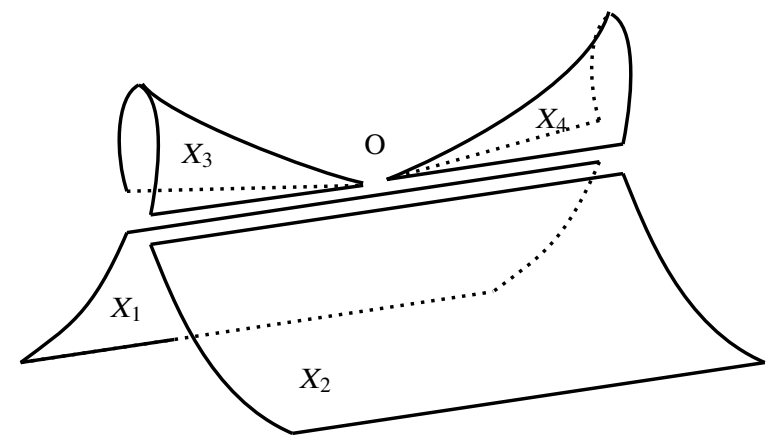

Fig. 4

[The term "generic" arises as follows. To prove existence of a regular stratification one proves that for an adjacent pair of strata $(X, Y)$,

$$
\{y \in Y \subset \bar{X}-X \mid(X, Y) \text { is regular at } y\}
$$

is generic in $Y$ in the Baire sense of containing a countable intersection of open dense subsets, so that its complement can be added to a closed set lower in the filtration than $\bar{Y}$.]

Theorem 1.6 Both $(a)$ and $(b)$ are $C^{1}$ invariants, i.e. given an (a)-regular (resp. (b)-regular) stratification of $Z \subset \mathbb{R}^{n}$ and a $C^{1}$ diffeomorphism $\phi: \mathbb{R}^{n} \longrightarrow \mathbb{R}^{n}$ then $\phi(Z)$ inherits an (a)-regular (resp. (b)-regular) stratification.

The previous result follows at once from the following characterizations of $(a)$ and $(b)$.

Let $\phi:(U, U \cap Y, y) \longrightarrow\left(\mathbb{R}^{n}, \mathbb{R}^{m} \times 0^{n-m}, 0\right)$ be a $C^{1}$ chart for $Y$ as a submanifold of $\mathbb{R}^{n}$. Let $\pi_{\phi}=\phi^{-1} \circ \pi_{m} \circ \phi: U \longrightarrow U \cap Y$ where $\pi_{m}: \mathbb{R}^{n} \longrightarrow \mathbb{R}^{m} \times 0^{n-m}$ is projection onto the first $m$ coordinates, and let $\rho_{\phi}=\rho_{m} \circ \phi: U \longrightarrow[0, \infty)$ where $\rho_{m}: \mathbb{R}^{n} \longrightarrow[0, \infty)$ is defined by $\rho_{m}\left(x_{1}, \ldots, x_{n}\right)=\sum_{i=m+1}^{n} x_{i}^{2}$.

First we characterize $(a)$-regularity.

Theorem 1.7 (Theorem A in [Tro79]) A pair of adjacent strata $(X, Y)$ is (a)-regular at $y \in Y \Longleftrightarrow$ for every $C^{1}$ foliation $\mathcal{F}$ transverse to $Y$ at $y$, there is a neighbourhood of y in which $\mathcal{F}$ is transverse to $X \Longleftrightarrow$ for every $C^{1} \operatorname{chart}(U, \phi)$ for $Y$ at $y$, there exists a neighbourhood $V$ of $y, V \subset U$, such that the retraction $\left.\pi_{\phi}\right|_{V \cap X}$ is a submersion.

Next we characterize (b)-regularity.

Theorem 1.8 ( Theorem B in [Tro79]) A pair of adjacent strata $(X, Y)$ is (b)-regular at $y \in Y \Longleftrightarrow$ for every $C^{1}$ chart $(U, \phi)$ for $Y$ at $y$, there is a neighbourhood $V$ of $y$, $V \subset U$, such that $\left.\left(\pi_{\phi}, \rho_{\phi}\right)\right|_{V \cap X}$ is a submersion. 


\subsection{The Kuo-Verdier condition (w)}

A natural idea is to seek stronger generic regularity conditions. Now Whitney's condition (a) says that

$$
\operatorname{dist}\left(T_{x} X, T_{y_{0}} Y\right) \longrightarrow 0 \text { as } x \rightarrow y_{0} .
$$

We can quantify this convergence in the stronger Kuo-Verdier condition [Ver76]

$$
\text { (w) } \quad \operatorname{dist}\left(T_{x} X, T_{y_{0}} Y=O\left(\left\|x-\pi_{Y}(x)\right\|=O(\operatorname{dist}(x, Y))\right.\right.
$$

i.e., there exists $C>0$, and a neighborhood $U$ of $y_{0}$ in $\mathbb{R}^{n}$ such that

$$
\operatorname{dist}\left(T_{x} X, T_{y_{0}} Y\right) \leq C\left\|x-\pi_{Y}(x)\right\| \forall x \in U \cap X .
$$

Here $\pi_{Y}$ denotes a $C^{1}$ submersive retraction from a tubular neighbourhood of $Y$ onto $Y$.

Theorem 1.9 Condition $(w)$ is generic, ie. (w)-regular stratifications exist in the various classes of sets.

See Verdier (Théorème 2.2 in [Ver76]), Denkowska and Wachta [DW87] or Lojasiewicz, Stasica and Wachta [LSW86] in the subanalytic case, and Tà Lê Loi [Loi98] for definable sets.

In Brodersen and Trotman ([BT79], Proposition 2) it was shown that condition $(w)$ can be characterized by lifting of vector fields. Precisely, $(w)$ holds for $(X, Y)$ at $y_{0} \in Y$ if and only if every vector field $v_{Y}$ on $Y$ extends in a neighborhood $U$ of $y_{0}$ to a vector field $v_{X}$ on $X$ which is rugose: $\exists C>0$ such that

$$
\forall x \in U \cap X, \forall y \in U \cap Y,\left\|v_{X}(x)-v_{Y}(y)\right\| \leq C\|x-y\| .
$$

Remark 1.10 The stratified vector field on $X \cup Y$ is weakly Lipschitz. For it to be Lipschitz one would need to impose the condition that

$$
\forall x \in U \cap X, \forall x^{\prime} \in U \cap X,\left\|v_{X}(x)-v_{X}\left(x^{\prime}\right)\right\| \leq C\left\|x-x^{\prime}\right\| .
$$

Theorem 1.11(1) For semi algebraic sets (also for subanalytic sets, and for definable sets in o-minimal structures), $(w)$ implies $(b)$.

(2) For complex analytic stratifications, $(w) \Longleftrightarrow(b)$.

For (1) in the subanalytic case see Kuo [Kuo71] or Verdier (Théorème 1.5 in [Ver76]). The definable case is due to Loi [Loi98]. (2) is due to Teissier (Théorème 1.2 in Chapter V of [Tei82]).

\section{Example.}

(vi) Let $V=\left\{y^{4}=t^{4} x+x^{3}\right\} \subset \mathbb{R}^{3}$, and stratify by $V \supset<O t>\supset \emptyset$. This satisfies $(b)$ but not $(w)$. In fact $V$ is a $C^{1}$ submanifold of $\mathbb{R}^{3}$, as proved in my thesis (Example 7.1 in [Tro77]). This shows that $(w)$ is not a $C^{1}$ invariant. 
One can check easily that condition $(w)$ is a $C^{2}$ invariant. In fact it is a $C^{1+\epsilon}$ invariant where the $\epsilon$ refers to a Hölder property of the first derivative. This fact is useful in proofs that $(w)$ is a generic condition.

\subsection{Mostowski's Lipschitz stratifications}

Mostowski [Mos85] introduced in 1985 a very strong regularity condition for complex analytic varieties and proved genericity. Then Parusiński successively proved genericity of Mostowski's Lipschitz condition for real analytic varieties [Par88b], for semi-analytic sets [Par88a] and finally for subanalytic sets [Par94]. Recently, N. Nguyen and Valette [NV16] proved genericity of Mostowski's Lipschitz condition for definable sets in polynomially bounded o-minimal structures.

Mostowski's original condition is rather technical and takes long to write down, so we will give an equivalent version due to Parusiński (Proposition 1.5 of [Par88a].

Definition 1.12 A stratification $\Sigma$ of a set $Z$ defined by

$$
Z=Z^{d} \supset Z^{d-1} \supset \cdots \supset Z^{0} \supset Z^{-1}=\emptyset
$$

is said to be a Lipschitz stratification (or satisfy condition $(L)$ ) if there exists a constant $K>0$ such that for every subset $W \subset Z$ such that

$$
Z^{j-1} \subseteq W \subseteq Z^{j}
$$

for some $j=\ell, \ldots, d$ where $\ell$ is the lowest dimension of a stratum of $\mathcal{Z}$, each Lipschitz $\Sigma$-compatible vector field on $W$ with Lipschitz constant $L$ which is bounded on $W \cap Z^{\ell}$ by a constant $C>0$, can be extended to a Lipschitz $\Sigma$-compatible vector field on $Z$ with Lipschitz constant $K(L+C)$.

Proposition 1.13 Every Lipschitz stratification satisfies condition (w).

This proposition is actually an immediate consequence of Mostowki's original definition [Mos85].

In fact, so far the Lipschitz condition is the strongest generic regularity condition on stratifications of definable sets.

\subsection{Applications of Whitney (a)-regularity}

We have been describing successively stronger regularity conditions. So, why should one study the rather weak Whitney $(a)$-regular stratifications ? One reason is because in singularity theory and dynamical systems (in classification problems and in the study of stability) one often uses that transversality to a Whitney stratification is an open condition. And in fact one can show the following equivalence, which gives 
another characterisation of $(a)$-regularity and hence another proof that $(a)$ is a $C^{1}$ invariant.

Theorem 1.14 (Theorem 1.1 in [Tro77], [Tro79]) Given a stratification $\Sigma$ of a closed subset $Z$ of a smooth manifold $M, \Sigma$ is Whitney (a)-regular $\Leftrightarrow\{f: N \longrightarrow$ $M \mid f$ is transverse to $\Sigma\}$ is an open set of $C^{1}(N, M)$ in the strong $C^{1}$ topology, for all $C^{1}$ manifolds $N$.

Recently, Trivedi gave holomorphic versions of this theorem for Stein manifolds $N, M[$ Tri13]

Another application of Whitney (a)-regularity is the following.

Theorem 1.15 (Kuo, Li and Trotman [KTL89]) Given a stratum $X$ of an (a)regular stratification of a subset $Z$ of $\mathbb{R}^{n}$, then for all $x \in X$ and for every pair of Lipschitz transversals $M_{1}, M_{2}$ to $X$ at $x$ (a Lipschitz transversal is defined to be the graph of a Lipschitz map $N_{x} X \rightarrow T_{x} X$ ), there is a homeomorphism

$$
\left(M_{1}, Z \cap M_{1}, x\right) \longrightarrow\left(M_{2}, Z \cap M_{2}, x\right) .
$$

These results justify the study and verification of (a)-regularity.

\section{Equisingularity}

We have seen in the examples how Whitney $(b)$-regularity allows us to distinguish points where the local topology changes. This is in fact a general property.

\subsection{Topological equisingularity}

Theorem 2.1 (Thom-Mather: Théorème 2.B.1 in [Tho69] and Proposition 11.1 in [Mat12]) $A$ Whitney (b)-regular stratification (of a closed subset $Z$ of a manifold $M)$ is locally topologically trivial along each stratum.

This means more precisely that for every point $x$ in a stratum $X$ there is a neighbourhood $U$ of $x$ in $M$, a stratified set $L$, and a stratified homeomorphism

$$
h:(U, U \cap Z, U \cap Z, x) \longrightarrow(U \cap X) \times\left(\mathbb{R}^{k}, c L, \star\right)
$$

such that $p_{1} \circ h=\pi_{X}$, where $c L$ denotes the cone on $L$ with vertex $\star$.

The proof of this theorem, known as the Thom-Mather first isotopy theorem, is by integration of a continuous stratified controlled vector field $v$ on $Z$ : for each stratum $X$, there is a lift of $v_{X}$ to a vector field $v_{Y}$ on neighbouring strata $Y$ such that $\pi_{X \star} v_{Y}=\pi_{X}$ and $\rho_{X \star} v_{Y}=0$ (these two conditions state that $v_{Y}$ is a lift of $v_{X}$ and that $v_{Y}$ is tangent to the level hypersurfaces of $\rho_{Y}$ ). 
In particular the isotopy theorem states that the local topological type of $Z$ at points of a stratum $X$ is locally constant, hence constant, as $X$ is connected.

Remark 2.2 That the lifted stratified vector field $v_{X} \cup v_{Y}$ in the Thom-Mather isotopy theorem can be chosen to be continuous was first independently proved by Shiota (Lemma I.1.5 in [Shi97]) and du Plessis [dP99]. A much stronger statement was recently proved as part of Whitney's fibering conjecture (Conjecture 9.2 in [Whi65b]). From the statement of the Thom-Mather theorem one can see that $h$ defines a foliation by leaves $h^{-1}(p)$ for $p \in c L$, each diffeomorphic to $U \cap X$. In the complex holomorphic case Whitney conjectured that the leaves be holomorphic and that their tangents vary continuously as we take the limit for points on a stratum $Y$ tending to an adjacent stratum $X$. This was proved by Parusiński and Paunescu (Theorem 7.6 in [PP17]) in the real and complex algebraic and analytic cases, using a hypothesis of a stratification which is Zariski equisingular (in a generic sense), stronger than (w)-regularity. In 2018 Parusinski has announced that this generic Zariski equisingularity implies the Lipschitz regularity of Mostowski for families of hypersurfaces in $\mathbb{C}^{3}$.

With the hypothesis of $(b)$-regularity (in fact with the even weaker $(c)$-regularity defined in Lecture III), Whitney's fibering conjecture was proved in the smooth case in 2017 by Murolo, du Plessis and Trotman (Theorem 7 in [MdPT17]): the leaves of $\left\{h^{-1}(p)\right\}_{p \in c L}$ form a $C^{0,1}$ foliation.

We saw above that a Kuo-Verdier ( $w$ )-regular stratification admits locally rugose vector fields tangent to strata. These may be integrated to provide a local rugose trivialization.

Theorem 2.3 (Verdier: Théorème 4.14 in [Ver76]) Every (w)-regular stratification is locally rugosely trivial along strata.

This is to say that a homeomorphism defining a trivialization (almost) as in the Thom-Mather theorem can be chosen to be rugose. This requires two clarifications. Firstly the homeomorphism of the Thom-Mather theorem is in fact already rugose because it is controlled - $h$ can be chosen to respect the level hyper surfaces of the control function $\rho_{X}$. Secondly in Verdier's theorem [Ver76] the homeomorphism is not in general with the product of $U \cap X$ and a cone, but rather with a normal slice see the counterexample using the topologist's sine curve below (Example 2.10).

Because of the definition we gave above of a Lipschitz stratification (of Mostowski) it is no surprise that there is also a local trivialization theorem for Lipschitz stratifications.

Theorem 2.4 (Mostowski [Mos85], Parusiński (Theorem 1.6 in [Par94])) Every Lipschitz stratification is locally bilipschitz trivial along strata.

Corollary 2.5 Every semialgebraic/subanalytic/definable subset of $\mathbb{R}^{n}$ admits a locally bilipschitz trivial stratification. 
Remark 2.6 Here "definable" must be taken in a polynomially bounded o-minimal structure: this means that every definable function $f: \mathbb{R}^{n} \longrightarrow \mathbb{R}$ satisfies $|f(x)| \leq$ $C\|x\|^{k}$, for some $C>0$ and some positive integer $k$, in a neighbourhood of infinity (i.e. outside some compact set $K \subset \mathbb{R}^{n}$ ).

Example 2.7 (Parusiński) Let $X(t)$ be $<O x>\cup\left\{\left(x, x^{t}, t\right) \mid x>0\right\} \subset \mathbb{R}^{3}$. Then the Lipschitz types of the $X(t)$ are all distinct for $t>1$. Hence there is no locally bilipschitz trivial stratification of $\cup X(t)$, thus no Lipschitz stratification.

This example is definable in any o-minimal structure which is not polynomially bounded $\left(x^{t}=\exp (\operatorname{tog} x)\right)$. Recall the theorem of C. Miller.

Theorem 2.8 (Miller [Mil94]) An o-minimal structure is not polynomially bounded if and only if the exponential function is definable in the structure.

Remark 2.9 When working outside of the class of definable sets, in the local triviality theorems for $(w)$-regular and Lipschitz stratifications we must replace $c L$ by a normal slice $F$ (not necessarily a cone), as shown by the following example.

Example 2.10 Let $Z=\overline{\{y=\sin (1 / x), x \neq 0\}} \subset \mathbb{R}^{2}$, the topologist's sine curve. If $Y=(-1,1) \times 0$ and $X=Z-Y$, with $(-1,0)$ and $(1,0)$ the 0 -strata, then we obtain a $(w)$ regular stratification and a Lipschitz stratification, but not a $(b)$-regular stratification. The stratification is locally topologically trivial indeed locally bilipschitz trivial along $Y$ but is not locally topologically conical. It is clear that $Z$ is not definable in an o-minimal structure because the $x$-axis intersects $Z$ in an infinite number of connected components.

Although local bilipschitz triviality is in general strictly weaker than the Lipschitz property of Mostowski, there exist (w)-regular stratified sets which are not locally bilipschitz trivial.

Example 2.11 (Koike). Let $Z=\left\{y^{2}=t^{2} x^{2}-x^{3}, x \leq 0\right\}$. This is obtained by removing the "upper half" of the Whitney cusp $V=\left\{y^{2}=t^{2} x^{2}-x^{3}\right\}$ (Figure 5).

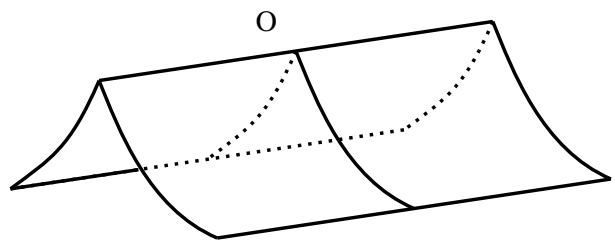

Fig. 5

Because the slices $t=$ constant vary between half of a double point, with a nonzero angle between the 2 branches, and a cusp, with zero angle between the 2 branches, one sees easily that these 2 types of slices are not bilipschitz equivalent. However the following calculation shows that $(w)$-regularity holds. 


$$
\begin{gathered}
d\left(<0 t>, T_{p} X\right)=\left\|<(0,0,1), \frac{\operatorname{grad}_{p} F}{\left\|\operatorname{grad}_{p} F\right\|}\right\| \\
=\frac{2\left|t x^{2}\right|}{\sqrt{\left(3 x^{2}-2 x t^{2}\right)^{2}+4 y^{2}+4 t^{2} x^{4}}} \\
\leq \frac{2\left|t x^{2}\right|}{2 y} \leq|x| \leq \sqrt{x^{2}+y^{2}}=\|p-\pi(p)\| .
\end{gathered}
$$

Thus (w) holds.

\subsection{Some complex equisingularity and real analogues}

We have seen that $(b)$-regularity implies the constance of the local topological type of a stratified set along each stratum. For families of complex plane curves defined by

$$
F: \mathbb{C}^{2} \times \mathbb{C} \longrightarrow \mathbb{C}, 0
$$

$\left(F^{-1}(0), 0 \times \mathbb{C}\right)$ is $(b)$-regular if and only if the local topological type of $F_{t}^{-1}(0)$ is constant as $t$ varies, where $(z, t)$ are the coordinates of $\mathbb{C}^{2} \times \mathbb{C}$. However this equivalence does not extend to higher dimensions as shown by the following celebrated example.

Example 2.12 (Briançon and Speder [BS75]). Let $F(x, y, z, t)=x^{3}+t x y^{3}+y^{4} z+z^{9}$. Then $\left(F^{-1}(0), 0 \times \mathbb{C}\right)$ is not $(b)$-regular at $(0,0,0,0)$, but the local topological type at $(0,0,0, t)$ of $\left.F_{t}\right) 1(0)$ is constant.

The theory of equisingularity aims at comparing different notions of regularity on stratifications, in particular of analytic varieties (where much work has been done in particular by Zariski, Teissier and Gaffney).

A basic invariant in algebraic geometry is the multiplicity $m_{0}(V)$ at a point 0 of a variety $V$ in $\mathbb{C}^{n}$. An informal definition of $m_{0}(V)$ is the number of points near 0 in $P \cap V$ for a generic plane $P$ of dimension equal to the codimension of $V$, passing near 0 .

A relation with stratifications is given by a theorem of Hironaka.

Theorem 2.13 (Hironaka 1969 (Corollary 6.2 in [Hir69])) Given a complex analytic Whitney (b)-regular stratification of a complex analytic variety $V$, the multiplicity of $V$ at points of $V$ is constant on strata.

Thus (b) implies equimultiplicity.

The proof is by integration of a vector field, and works for subanalytic sets, interpreted as $(b)$ implying normal pseudo flatness (this is equivalent to equimultiplicity in the complex case), as shown in a paper of mine with Orro (Proposition 5.2 in [OT02]). (One defines the normal cone of a stratified set along a stratum $X$ by taking 
limits on $X$ of orthogonal secant vectors from $Y$ to the set and then normal pseudo flatness means that the associated projection of the normal cone to $X$ is open.)

\subsubsection{Zariski's problem}

In 1971, Zariski stated the following problem (Question A in [Zar71]): Given analytic functions $f, g: \mathbb{C}^{n+1}, 0 \longrightarrow \mathbb{C}, 0$ and a germ at 0 of a homeomorphism $h$ of $\mathbb{C}^{n+1}$ sending $f^{-1}(0)$ onto $g^{-1}(0)$, does $m_{0}\left(f^{-1}(0)\right)=m_{0}\left(g^{-1}(0)\right)$ ?

As this school concerns the Lipschitz geometry of singularities I will mention some results about Zariski's problem when the homeomorphism $h$ is assumed to be bilipschitz.

Theorem 2.14 (Fernandes and Sampaio [FS16]) Zariski's problem has a positive answer if $n=2$ and $h$ is bilipschitz.

Theorem 2.15 (Risler and Trotman [RT97]) Zariski's problem has a positive answer if $h$ is bilipschitz and $f=g \circ h$, for all $n$.

In 2018 it was announced by Birbrair, Fernandes, Sampaio and Verbitsky [BFSV18] that for the non hypersurface case there are infinitely many counterexamples to the bilipschitz invariance of the multiplicity with the dimension of the varieties being at least 3 .

For normal complex surfaces (possibly embedded in higher dimensions), Neumann and Pichon [NP12] have proved that the multiplicity is an outer bilipschitz invariant.

Theorem 2.16 (Comte [Com98]) Zariski's problem has a positive answer for complex analytic germs if $h$ is bilipschitz with Lipschitz constants (of $h$ and $h^{-1}$ ) sufficiently close to 1 .

More precisely if $X_{1}$ and $X_{2}$ are complex analytic germs of dimension $d$ in $\mathbb{C}^{n}$ and there exist constants $C>0, C^{\prime}>0$ such that

$$
\left(1 / C^{\prime}\right)\|x-y\| \leq\|h(x)-h(y)\| \leq C\|x-y\|
$$

for all $x, y$ near 0 in $X_{1}$ for a bilipschitz homeomorphism $h: X_{1}, 0 \longrightarrow X_{2}, 0$ and

$$
1 \leq C C^{\prime} \leq\left(1+\frac{1}{M}\right)^{\frac{1}{2 d}}
$$

where $M=\max \left(m_{0}\left(X_{1}\right), m_{0}\left(X_{2}\right)\right)$, then $m_{0}\left(X_{1}\right)=m_{0}\left(X_{2}\right)$.

The proof uses a characterization of the multiplicity as the density, originally due to Lelong [Lel57].

Definition 2.17 The density of a set $X$ at $p \in X$ is defined as the limit as $r$ tends to 0 of the volume of the intersection of $X$ with the ball of radius $r$ centred at $p$ divided by the volume of the intersection of a plane through $p$ of the same dimension as $X$ with the ball of radius $r$ centred at $p$. 
Corollary 2.18 (Comte [Com98]) In a bilipschitz trivial family of complex analytic germs (defined by a Lipschitz isotopy) the multiplicity is constant.

While on the topic of equimultiplicity and stratifications one should mention the important characterization due to Teissier.

Theorem 2.19 (Teissier: Théorème 1.2 in Chapter $\mathbf{V}$ of [Tei82]) A complex analytic stratification of a complex analytic variety is Whitney $(b)$-regular $\Longleftrightarrow$ the multiplicities of the local polar varieties are constant on strata.

Here the local polar varieties at a point of the variety are the closures of the critical sets of the restrictions to strata, whose closure contains the point, of locally defined projections to general linear subspaces of dimensions lying between two and the dimension of the variety (see section 3.2 in [FT]).

There are real analogues of these complex results involving what are known as Lipschitz-Killing invariants on strata of a definable stratification, due to Comte and Merle [CM08] and Nguyen and Valette [NV18]. These generalize another real analogue of Hironaka's theorem stated above, due to Comte (who proved in 2000 the partial result (Théorème 0.4 of [Com00]) of continuity of the density along strata of a (w)-regular subanalytic stratification) and G. Valette.

Theorem 2.20 (Valette [Val08]) The density is a Lipschitz function along strata of $a(w)$-regular subanalytic stratification, and a continuous function along strata of a (b)-regular subanalytic stratification.

Part of the proof of Teissier's theorem 2.19 above involves studying how equisingularity is preserved after taking generic plane sections of different dimensions. Precisely, let $Y \subset \bar{X}-X$.

Definition 2.21 Consider a plane $P \supset Y$, then $(X \cap P, Y)$ is a stratified pair. If $E$ is an equisingularity condition, such as $(b)$ or $(w)$, etc., then one says that the pair $(X, Y)$ is $E^{\star}$-regular at $0 \in Y$ if for all $k, 0 \leq k \leq n-m$ there exists an open dense set of planes $P$ of codimension $k$ such that $P$ is transverse to $X$ near 0 and $(X \cap P, Y)$ is $E$-regular at 0 .

If we abbreviate local topological triviality by (T.T.) then Teissier proved in the complex case that $(b)$ implies $\left(T . T .^{\star}\right)$, a strengthening of the Thom-Mather theorem, while the converse, that $\left(T . T .^{\star}\right)$ implies $(b)$, was proved by Lê and Teissier (see Théorème 5.3.1 in [LT83]). This is thus a converse to Teissier's strengthened Thom-Mather theorem. Next we give some results concerning the ${ }^{\star}$ condition in the subanalytic case.

Theorem 2.22 For subanalytic stratifications, $(w)$ implies $\left(w^{\star}\right)$, the Lipschitz property $(L)$ implies $\left(L^{\star}\right)$ and, when $Y$ has dimension $1,(b)$ implies $\left(b^{\star}\right)$.

The first and third implications are proved by Navarro and Trotman (Theorem 3.14 in [NAT81]), and the second implication is proved by Juniati, Trotman and Valette (Corollary 2.9 in [JTV03]). It is unknown if $(b)$ implies $\left(b^{\star}\right)$ when the dimension of 
$Y$ is greater than 1 for subanalytic stratifications, but in the complex case this follows from the implication that $(w)$ implies $\left(w^{\star}\right)$ since $(b)$ and $(w)$ are equivalent (Teissier: Théorème 1.2 in Chapter V of [Tei82]). Probably the implications in the previous theorem are valid for definable sets in polynomially bounded o-minimal structures. A counterexample to the third implication in the non polynomially bounded case is given in a paper by myself and Valette (in section 4 of [TV17]). Another such example is given in a paper by myself and L. Wilson [TW06].

Example 2.23 (Trotman and Wilson [TW06]) Let $f(x, z)=z-\frac{z \log \left(x+\sqrt{x^{2}+z^{2}}\right.}{\log z}, z>0$. Then let $S_{f}$ be the closure of the graph of $f$ in $\mathbb{R}^{3}$. Then $\left(S_{f}-<O x>,<0 x>\right.$ ) is $(b)$-regular, but $\left(b^{\star}\right)$ fails. Also $(w)$ fails to hold, and normal pseudo flatness fails.

Example 2.24 (Trotman and Valette (section 4 in [TV17])) Let

$$
g(x, z)=z^{x^{2}+1}=\exp \left(\left(x^{2}+1\right) \log z\right), z>0 .
$$

(Figure 6) Let $S_{g}$ be the closure of the graph of $g$ in $\mathbb{R}^{3}$. Then $\left(S_{g}-<0 x>,<0 x>\right.$ ) is $(b)$-regular, but $\left(b^{\star}\right)$ fails. Also normal pseudo flatness and $(w)$ fail to hold.

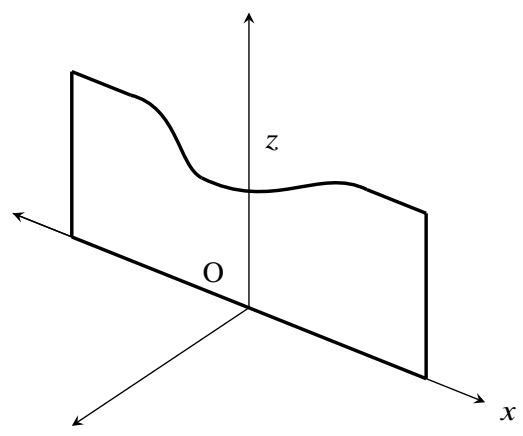

Fig. 6: Page 14, Example 2.24

Consider the convex hull $K_{g}$ of $S_{g}$ and the half-plane $\{y=0, z>0\}$. The density of $K_{g}$ is not continuous along $0 x$ at 0 . This is a counterexample to a possible generalization of the Comte-Valette theorem 2.20 ([Com00], [Val08]) to non polynomially bounded o-minimal structures. Note too that the bilipschitz type of $K_{g}$ varies continuously along $0 x$.

These two examples provide examples of definable sets in any non polynomially bounded o-minimal structure, because in such a structure the exponential function and its logarithm inverse are definable by Miller's dichotomy [Mil94] stated above.

These examples prevent definable extensions to the following theorem.

Theorem 2.25 (Pawłucki (Theorem 1.1 in [Paw85])) Let $X, Y$ be (locally) connected subanalytic strata in $\mathbb{R}^{n}, Y \subset \bar{X}-X$, such that $\operatorname{dim} X=\operatorname{dim} Y+1$. Then $(X, Y)$ is (b)-regular $\Longleftrightarrow X \cup Y$ is a $C^{1}$ manifold-with-boundary. 
One implication is just the $C^{1}$ invariance of $(b)$. The other is more delicate.

In 2017 with Valette (Corollary 3.11 in [TV17]) I proved that Pawłucki's characterization is valid for definable sets in polynomially bounded o-minimal structures.

The two examples above show this fails in non polynomially bounded o-minimal structures.

\section{Triangulation of stratified sets and maps}

While stratifications can be thought of as a more efficient alternative to triangulations, as there are less strata in a stratification into manifolds than simplexes of maximal dimension in a triangulation, it remains the case that triangulations of sets (and maps) are useful for calculating homology and cohomology. In this section we present results concerning sets and maps, rather incomplete, but which may serve as an introduction to the theory.

\subsection{Triangulation of sets}

Theorem 3.1 (Hironaka [Hir75]) Every semialgebraic set $S$ is triangulable : there exists a polyhedron $K$ and a semialgebraic homeomorphism $\phi: K \longrightarrow S$. Moreover given a finite family $\left\{S_{j}\right\}_{j=1, \ldots, m}$ of semialgebraic subsets of $S$, we can choose $K=\left\{\sigma_{i}\right\}_{I=1, \ldots, p}$ (the simplexes) and $\phi$ such that each $S_{j}$ is the union of some of the $\phi\left(\sigma_{i}^{o}\right)$.

The proof applies also to the case of subanalytic sets.

Corollary 3.2 A Whitney stratified semialgebraic set $S$ admits a triangulation such that every stratum is a union of (images of) open simplexes.

There are similar results in the smooth category.

Theorem 3.3 (Goresky [Gor78]) Every Whitney stratified set is triangulable, such that strata are unions of open simplexes.

Conjecture 3.4 (Thom) Every Whitney stratified set admits a Whitney triangulation, i.e. a triangulation such that the refined stratification defined by the open simplexes is itself $(b)$-regular.

Theorem 3.5 (Murolo and Trotman [MdPT19]) Every Whitney stratified set admits a Whitney cellulation, i.e. a cellular decomposition such that the refinement of the stratification by the open cells is (b)-regular.

Corollary 3.6 (A conjecture of Goresky [Gor81]). The homology of a Whitney stratified set $X$ can be represented by Whitney stratified cycles $\left(W H_{\star}(X) \cong H_{\star}(X)\right)$. 
As a partial answer to Thom's conjecture we have the following result of Shiota in the semialgebraic case.

Theorem 3.7 (Shiota [Shi05]) Every semialgebraic set $S$ admits a semialgebraic Whitney triangulation, i.e. the open simplexes $\phi\left(\sigma_{i}^{o}\right)$ form the strata of a Whitney stratification, and this may be chosen to be compatible with a finite set of semialgebraic subsets of $S$.

Shiota's theorem was improved and extended by Malgorzata Czapla in her thesis.

Theorem 3.8 (Czapla [Cza12]) Every definable set $S$ admits a definable $C^{2}(w)$ regular triangulation, compatible with a finite number of definable subsets of $S$. Moreover the triangulation $\phi:|K| \longrightarrow S$ is a locally Lipschitz mapping.

So Czapla improves on Shiota's theorem in two ways: $(w)$-regularity and definability. The main tool of Czapla is a bilipschitz triviality theorem of Valette for definable families, itself an improvement of a celebrated theorem of Hardt.

A continuous semialgebraic mapping $p: A \longrightarrow \mathbb{R}^{k}$ where $A \subset \mathbb{R}^{n}$ is semialgebraic, is said to be semialgebraically trivial over a semialgebraic subset $B \subset \mathbb{R}^{k}$ if there is a semialgebraic set $F$ and a semialgebraic homeomorphism $h: p^{-1}(B) \longrightarrow B \times F$ such that $p_{1} \circ h=p$. Then $h$ is called a semialgebraic trivialization of $p$ over $B$. We say $h$ is compatible with $C \subset A$ if there exists a semialgebraic set $G \subset F$ such that $h\left(C \cap p^{-1}(B)\right)=B \times G$.

Theorem 3.9 (Hardt's semialgebraic triviality [Har80]) Let $A \subset \mathbb{R}^{n}$ be a semialgebraic set and $p: A \longrightarrow \mathbb{R}^{k}$ a continuous semialgebraic mapping. Then there is a finite semialgebraic partition of $\mathbb{R}^{k}$ into $B_{1}, \ldots, B_{m}$ such that $p$ is semi algebraically trivial over each $B_{i}$. Moreover if $C_{1}, \ldots, C_{q}$ are semialgebraic subsets of $A$ we can assure that each trivialization

$$
h_{i}: p^{-1}\left(B_{i}\right) \longrightarrow B_{i} \times F_{i}
$$

is compatible with all $C_{j}$.

In particular if $b, b^{\prime} \in B_{i}$, then $p^{-1}(b)$ and $p^{-1}\left(b^{\prime}\right)$ are semialgebraically homeomorphic. One can take $F_{i}=p^{-1}\left(b_{i}\right), b_{i} \in B_{i}$ and set $h_{i}(x)=\left(x, b_{i}\right)$ for all $x \in p^{-1}\left(b_{i}\right)$.

There is a definable version of Hardt's triviality theorem too, given by Coste in his Pisa notes on semialgebraic geometry [Cos00]. We now consider a further improvement, a definable bilipschitz triviality theorem due to G. Valette [Val05a].

Fix a polynomially bounded o-minimal structure over $\mathbb{R}$ (take semialgebraic sets if preferred). Let $A \subset \mathbb{R}^{n} \times \mathbb{R}^{p}$ be a definable set, considered as a family of definable subsets of $\mathbb{R}^{n}$ parametrized by $\mathbb{R}^{p}$. For $U \subset \mathbb{R}^{p}$, let

$$
A_{U}=\left\{q=(x, t) \in \mathbb{R}^{n} \times \mathbb{R}^{p} \mid q \in A, t \in U\right\}
$$

and for $t \in \mathbb{R}^{p}$, let $A_{t}=\left\{x \in \mathbb{R}^{n} \mid q=(x, t) \in A\right\}$, the fibre of $A$ at $t$. 
Definition 3.10 $A$ is said to be definably bilipschitz trivial along $U \subseteq \mathbb{R}^{p}$ if there exists $t_{0} \in U$ and a definable homeomorphism $h: A_{t_{0}} \times U \longrightarrow A_{U}$ mapping $(x, t)$ to $h(x, t)=\left(h_{t}(x), t\right)$ together with a definable continuous function $C: U \longrightarrow \mathbb{R}$ such that for all $x, x^{\prime} \in A_{t_{0}}$ and all $t \in U$,

$$
\left|h_{t}(x)-h_{t}\left(x^{\prime}\right)\right| \leq C(t)\left|x-x^{\prime}\right|
$$

and for all $x, x^{\prime} \in A_{t}$, and all $t \in U$,

$$
\left|h_{t}^{-1}(x)-h_{t}^{-1}\left(x^{\prime}\right)\right| \leq C(t)\left|x-x^{\prime}\right| .
$$

Theorem 3.11 (Valette [Val05a, Val05b]) Let $A$ be a definable subset of $\mathbb{R}^{n} \times \mathbb{R}^{p}$ in some polynomially bounded structure over $\mathbb{R}$. Then there exists a definable partition of $\mathbb{R}^{p}$ such that the family $A$ is definably bilipschitz trivial along each element of the partition.

Notes. The Mostowski-Parusiński condition $(L)$ of section 1.3 together with the definable existence theorem of Nguyen and Valette [NV16] gives a local bilipschitz trivialization $h$. Here we have definability of $h$ as well. There is also better control of the Lipschitz constants of the bilipschitz trivialization here.

As in the case of Hardt's theorem for topological types we can deduce from Valette's theorem bounds on the number of Lipschitz types of sets given as zeros of polynomials of bounded degree.

To prove his theorem, Valette proves a preparation theorem, and uses ultrafilters as in Coste's account of the definable Hardt triviality theorem (cf. Coste's Pisa notes on o-minimal geometry [Cos00]).

\subsection{Thom maps and the $\left(a_{f}\right)$ condition}

We will describe a class of stratified maps which are triangulable.

Definition 3.12 Let $Z$ be a closed subset of $\mathbb{R}^{n}\left(\right.$ or $\mathbb{C}^{n}$ ) with a stratification $\Sigma$. Let $f: \mathbb{R}^{n} \longrightarrow \mathbb{R}^{p}$ be a $C^{1}$ map. Then $\Sigma$ is said to satisfy $\left(a_{f}\right)$ if each $\left.f\right|_{X}$, for $X$ a stratum of $\Sigma$, is of constant rank (depending on $X$ ), and for sequences $x_{i} \in X$ tending to $y$ in a stratum $Y$ of $\Sigma$,

$$
\lim _{x_{i} \rightarrow y} T_{x_{i}}\left(f^{-1}\left(f\left(x_{i}\right)\right)\right) \supseteq T_{y}\left(f^{-1}(f(y))\right) .
$$

When further,

$$
\operatorname{dist}\left(T_{x}\left(f^{-1}(f(x))\right), T_{y}\left(f^{-1}(f(y))\right) \leq C|| x-\pi_{Y}(x) \|\right.
$$

for some $C>0$ and $x$ in a neighbourhood $U$ of $y$ in $\mathbb{R}^{n}$ (or $\mathbb{C}^{n}$ ), we say that $\Sigma$ satisfies the $\left(w_{f}\right)$ condition. 
Theorem 3.13 (Loi [Loi98]) For polynomially bounded o-minimal structures, every definable function $f: \mathbb{R}^{n} \longrightarrow \mathbb{R}$ admits a stratification such that $\left(w_{f}\right)$ holds.

For $\left(a_{f}\right)$ this is true in any o-minimal structure. In the complex case the result is due to Henry, Merle and Sabbah [HMS84].

Definition 3.14 Let $f: \mathbb{R}^{n} \longrightarrow \mathbb{R}^{m}$ be a $C^{1}$ map. If there exist Whitney stratifications $\Sigma$ of $Z \subset \mathbb{R}^{n} \subset \mathbb{R}^{m}$ such that $f$ maps each stratum $X$ of $\Sigma$ to a stratum $X^{\prime}$ of $\Sigma^{\prime}$, such that $\left.f\right|_{X}$ is a submersion onto $X^{\prime}, \Sigma$ satisfies $\left(a_{f}\right)$, and each $\left.f\right|_{X}$ is proper, then one says that $f$ is a Thom map .

Thom maps have nice properties.

Theorem 3.15 (Shiota [Shi00]) If $Z, W$ are respectively closed subsets of $\mathbb{R}^{n}$ and $\mathbb{R}^{m}$ and $f: Z \longrightarrow W$ is a proper $C^{\infty}$ Thom map, then $f$ is triangulable, i.e. there exist polyhedra $P, Q$ and homeomorphisms $\phi: Z \longrightarrow P, \psi: W \longrightarrow Q$ such that $\psi \circ f \circ \phi^{-1}: P \longrightarrow Q$ is piecewise linear.

For non-proper maps there is still a theorem.

Theorem 3.16 (Shiota [Shi10]) Nonproper semialgebraic $C^{1}$ Thom maps between closed semialgebraic subsets are triangulable, i.e. there exist finite simplicial complexes $K, L$ and semialgebraic (resp. definable) $C^{0}$ embeddings $\phi: Z \longrightarrow|K|$, $\psi: W \longrightarrow|L|$ such that $\phi(Z)$ and $\psi(W)$ are unions of open simplexes of $K, L$ and $\psi \circ f \circ \phi^{-1}: \phi(Z) \longrightarrow \psi(W)$ can be extended to a simplicial map $K \longrightarrow L$.

When the target space is of dimension $>1$, the transform of the map by suitable blowing-ups of the target space becomes $\left(a_{f}\right)$ stratifiable (see Sabbah [Sab83]) and locally triangulable (see Teissier [Tei89]). Note that maps not satisfying $\left(a_{f}\right)$ may not be triangulable. For example the blowup of a point in $\mathbb{R}^{2}$ does not satisfy $\left(a_{f}\right)$ and is not triangulable. Any 2-simplex attached to the exceptional fibre (a projective line) is mapped to a 1-simplex by linearity. One sees that $\left(a_{f}\right)$ fails because outside the origin the fibres of points are just points and the limit of a point cannot contain a line as the tangent space of the exceptional fibre. Thom [Tho69] called maps satisfying $\left(a_{f}\right)$ maps "sans éclatement", i.e. without blowing-up, so that this example is in some sense a paradigm.

Analogous to the characterization of (a)-regularity by the openness of the set of maps transverse to a stratification, we have a similar result for $\left(a_{f}\right)$-maps.

Theorem 3.17 (Trivedi-Trotman [TT14]) Let $N, P$ be $C^{1}$ manifolds. Let $f: N \longrightarrow$ $P$ be a $C^{1}$ map of constant rank on the strata of a stratification $\Sigma$ of a closed subset $Z$ of $N$. Let $\mathcal{F}$ denote the foliations of strata $X$ of $\Sigma$ induced by the fibers of $\left.f\right|_{X}$. The following are equivalent:

(1) $\Sigma$ is $\left(a_{f}\right)$-regular;

(2) for any $C^{1}$ manifold $M,\left\{g \in C^{1}(M, N): g\right.$ is transverse to $\left.\mathcal{F}\right\}$ is open in the strong $C^{1}$ topology;

(3) $\left\{g \in C^{1}(N, N): g\right.$ is transverse to $\left.\mathcal{F}\right\}$ is open in the strong $C^{1}$ topology. 
The $\left(a_{f}\right)$ condition has a particular role in equisingularity of families of complex hypersurfaces.

Let $F=\mathbb{C}^{n+1} \times \mathbb{C}, O \times \mathbb{C} \longrightarrow \mathbb{C}, 0$ ba an analytic function such that the singular locus of $F^{-1}(0)$ is $0 \times \mathbb{C}$. Let $F_{t}(z)=F(z, t)$.

Theorem 3.18 (Lê and Saito [LS73], Teissier (Remarque 3.10 in [Tei73])) . The following conditions are equivalent:

(1) $\mu\left(F_{t}\right)$ is constant as $t$ varies,

(2) $\left(a_{f}\right)$ holds for the stratification $\left(F^{-1}(0)-0 \times \mathbb{C}, 0 \times \mathbb{C}\right)$,

(3) $\lim _{(z, t) \rightarrow(0,0} \frac{|\partial F / \partial t|}{|\operatorname{gradF}|}=0$.

Corollary 3.19 If $F(z, t)=g(z)+\operatorname{th}(z)$ has $\mu\left(F_{t}\right)$ constant, then $F_{t}$ is equimultiple along $0 \times \mathbb{C}$.

This simple consequence of the previous theorem should be linked to a striking result of Parusinski.

Theorem 3.20 (Parusiński [Par99]) With the same hypotheses as in the previous corollary, the topological type of $F_{t}^{-1}(0)$ is constant as $t$ varies.

This in turn should make us think again of an important general result.

Theorem 3.21 (Lê-Ramanujam [LR76]). If $n \neq 2$ and $\mu\left(F_{t}\right)$ is constant, then the topological type of $F_{t}^{-1}(0)$ is constant.

Question. What happens when $n=2$ ?

Remark 3.22 There are at least 3 different definitions of $\left(b_{f}\right)$-regularity, due to Thom (in section IIIB of [Tho69]), Henry-Merle (Definition 9.1.1 of [HM87]), and Nakai (see $\S 1$ of [Nak00]). Their properties have not been studied beyond the original papers so far as I know. And no work has been done on a possible $\left(L_{f}\right)$.

\subsection{1 (c)-regularity}

The notion of Thom map has been used by Karim Bekka to define a new regularity condition called $(c)$.

Definition 3.23 One says that a stratification $\Sigma$ of a closed set $Z$ in a manifold $M$ is (c)-regular if for each stratum $X$ of $\Sigma$ there is a neighbourhood $U_{X}$ of $X$ in $M$ and a $C^{1}$ function $\rho_{X}: U_{X} \longrightarrow[0,1)$ such that $X=\rho_{X}^{-1}(0)$ and $\rho_{X}$ is a Thom map for $\Sigma$.

One shows fairly easily that $(b) \Longrightarrow(c) \Longrightarrow(a)$. Note that $U_{X}$ is a neighbourhood of the whole of $X$ and not just of a point of $X$.

Moreover, by a careful analysis of the proof of the Thom-Mather isotopy theorem, Bekka showed: 
Theorem 3.24 (Bekka (see \$3 in [Bek91])) Every (c)-regular stratification is locally topologically trivial along strata (and conical).

Thus, as for Whitney (b)-regular stratified sets $(Z, \Sigma)$, for every point $x$ in a stratum $X$ there is a neighbourhood $U$ of $x$ in $M$, a stratified set $L$ and a homeomorphism

$$
h:(U, U \cap Z, U \cap X) \longrightarrow(U \cap X) \times\left(\mathbb{R}^{k}, c L, \star\right)
$$

given by $h(z)=\left(\pi_{X}(z), \rho_{X}(z), \theta(z)\right)$ where $c L$ is the cone on $L$ with vertex $\star$. As for $(b)$-regularity, fix the values of $\rho_{X}$ and $\theta$, then $\left\{z \mid \rho_{X}(z)=\rho, \theta(z)=\theta\right\}$ is a leaf diffeomorphic to $U \cap X$.

Theorem 3.25 (Murolo-du Plessis-Trotman (Theorem 7 in [MdPT17])) Given a (c)-regular stratified set we can choose $h$ such that the tangent spaces to the leaves vary continuously on $U$, in particular as points tend to $X$.

Again we may fix just $\theta$. Then $\{z \mid \theta(z)=\theta\}$ is a wing, a $C^{0}$ manifold with boundary $U \cap X$ and smooth interior. Then one can choose $h$ so that the tangent spaces to the wings vary continuously and each wing is itself (c)-regular (Theorem 8 in [MdPT17]).

Question. What can one say in the semialgebraic or subanalytic cases? Note that the Parusiński-Paunescu theorem (Theorem 7.6 of [PP17] ) is only for the algebraic and analytic cases.

\section{References}

Bek91. Karim Bekka. C-régularité et trivialité topologique. In Singularity theory and its applications, Part I (Coventry, 1988/1989), volume 1462 of Lecture Notes in Math., pages 42-62. Springer, Berlin, 1991. 20

BFSV18. Lev Birbrair, Alexandre Fernandes, Jose Edson Sampaio, and Misha Verbitsky. Multiplicity of singularities is not a bi-lipschitz invariant. arXiv preprint arXiv:1801.06849, 2018. Math. Annalen, to appear, 2020. 12

BS75. Joël Briançon and Jean-Paul Speder. La trivialité topologique n'implique pas les conditions de Whitney. C. R. Acad. Sci. Paris Sér. A-B, 280(6):Aiii, A365-A367, 1975. 11

BT79. Hans Brodersen and David Trotman. Whitney (b)-regularity is weaker than Kuo's ratio test for real algebraic stratifications. Math. Scand., 45(1):27-34, 1979. 6

CM08. Georges Comte and Michel Merle. Équisingularité réelle. II. Invariants locaux et conditions de régularité. Ann. Sci. Éc. Norm. Supér. (4), 41(2):221-269, 2008. 13

Com98. Georges Comte. Multiplicity of complex analytic sets and bi-Lipschitz maps. In Real analytic and algebraic singularities (Nagoya/Sapporo/Hachioji, 1996), volume 381 of Pitman Res. Notes Math. Ser., pages 182-188. Longman, Harlow, 1998. 12, 13

Com00. Georges Comte. Équisingularité réelle: nombres de Lelong et images polaires. Ann. Sci. École Norm. Sup. (4), 33(6):757-788, 2000. 13, 14

Cos00. Michel Coste. An Introduction to O-minimal Geometry. Dottorato di ricerca in matematica / Università di Pisa, Dipartimento di Matematica. Istituti editoriali e poligrafici internazionali, 2000. 16, 17 
Cza12. Małgorzata Czapla. Definable triangulations with regularity conditions. Geom. Topol., 16(4):2067-2095, 2012. 16

dP99. Andrew du Plessis. Continuous controlled vector fields. In Singularity theory (Liverpool, 1996), volume 263 of London Math. Soc. Lecture Note Ser, pages xviii-xix, 189-197. Cambridge Univ. Press, Cambridge, 1999. 9

DW87. Zofia Denkowska and Krystyna Wachta. Une construction de la stratification sousanalytique avec la condition (w). Bull. Polish Acad. Sci. Math., 35(7-8):401-405, 1987. 6

DWS85. Zofia Denkowska, Krystyna Wachta and Jacek Stasica. Stratification des ensembles sous-analytiques avec les propriétés (A) et (B) de Whitney. Univ. Iagel. Acta Math., 25:183-188, 1985. 4

FS16. Alexandre Fernandes and Jose Edson Sampaio. Multiplicity of analytic hypersurface singularities under bi-Lipschitz homeomorphisms. J. Topol., 9(3):927-933, 2016. 12

FT. Arturo Giles Flores and Bernard Teissier. Local polar varieties in the geometric study of singularities. Ann. Fac. Sci. Toulouse Math., (6) 27:679-775, 2018. 13

Gor78. Mark Goresky. Triangulation of stratified objects. Proc. Amer. Math. Soc., 72(1):193200, 1978. 15

Gor81. Mark Goresky. Whitney stratified chains and cochains. Trans. Amer. Math. Soc., 267(1):175-196, 1981. 15

Hal14a. Immanuel Halupczok Non-Archimedean Whitney stratifications. Proc. London Math. Soc. (3) 109:1304-1362, 2014. 4

Hal14b. Immanuel Halupczok Stratifications in valued fields. in Valuation theory in interaction, EMS Ser. Congr. Rep., Eur. Math. Soc., Zurich, pages 288-296, Z014. 4

Har75. Robert Hardt. Stratification of real analytic mappings and images. Invent. Math., 28:193-208, 1975. 4

Har80. Robert Hardt. Semi-algebraic local-triviality in semi-algebraic mappings. Amer. J. Math., 102(2):291-302, 1980. 16

Hir69. Heisuke Hironaka. Normal cones in analytic Whitney stratifications. Inst. Hautes Études Sci. Publ. Math., (36):127-138, 1969. 11

Hir73. Heisuke Hironaka. Subanalytic sets. In Number theory, algebraic geometry and commutative algebra, in honor of Yasuo Akizuki, pages 453-493. 1973. 4

Hir75. Heisuke Hironaka. Triangulations of algebraic sets. In Algebraic geometry (Proc. Sympos. Pure Math., Vol. 29, Humboldt State Univ., Arcata, Calif., 1974), pages 165185. Amer. Math. Soc., Providence, R.I., 1975. 15

HM87. Jean-Pierre Henry and Michel Merle. Conditions de régularité et éclatements. Ann. Inst. Fourier (Grenoble), 37(3):159-190, 1987. 19

HMS84. Jean-Pierre Henry and Michel Merle, and Claude Sabbah. Sur la condition de Thom stricte pour un morphisme analytique complexe. Ann. Sci. École Norm. Sup. (4), 17(2):227-268, 1984. 18

JTV03. Dwi Juniati, David Trotman, and Guillaume Valette. Lipschitz stratifications and generic wings. J. London Math. Soc. (2), 68(1):133-147, 2003. 13

Ka105. Vadim Yu. Kaloshin. A geometric proof of the existence of Whitney stratifications. Mosc. Math. J., 5(1):125-133, 2005. 4

KTL89. Tzee Char Kuo, and Pei Xin Li, and David J. A. Trotman, Blowing-up and Whitney (a)-regularity. Canad. Math. Bull., 32(4):482-485, 1989. 8

Kuo71. Tzee Char Kuo. The ratio test for analytic Whitney stratifications. In Proceedings of Liverpool Singularities-Symposium, I (1969/70), Lecture Notes in Mathematics, Vol. 192, pages 141-149. Springer, Berlin, 1971. 6

LR76. Lê Dũng Tráng and C. P. Ramanujam. The invariance of Milnor's number implies the invariance of the topological type. Amer. J. Math., 98(1):67-78, 1976. 19

LS73. Lê Dũng Tráng and Kyoji Saito. La constance du nombre de Milnor donne des bonnes stratifications. C. R. Acad. Sci. Paris Sér. A-B, 277:A793-A795, 1973. 19

LT83. Lê Dũng Tráng and Bernard Teissier. Cycles evanescents, sections planes et conditions de Whitney. II. In Singularities, Part 2 (Arcata, Calif., 1981), volume 40 of Proc. Sympos. Pure Math., pages 65-103. Amer. Math. Soc., Providence, RI, 1983. 13 
Lel57. Pierre Lelong. Intégration sur un ensemble analytique complexe. Bull. Soc. Math. France, 85:239-262, 1957. 12

Loi98. Ta Lê Loi. Verdier and strict Thom stratifications in o-minimal structures. Illinois J. Math., 42(2):347-356, 1998. 4, 6, 18

Loj65. Stanisław Łojasiewicz. Ensembles semi-analytiques. I.H.E.S.notes, 1965. 4

LSW86. Stanisław Lojasiewicz, Jacek Stasica, and Krystyna Wachta. Stratifications sousanalytiques. Condition de Verdier. Bull. Polish Acad. Sci. Math., 34(9-10):531-539 (1987), 1986. 6

Mat12. John Mather. Notes on topological stability. Bull. Amer. Math. Soc. (N.S.), 49(4):475506, 2012. 4, 8

MdPT17. Claudio Murolo, Andrew du Plessis, and David J. A. Trotman. On the smooth whitney fibering conjecture. hal preprint hal-01571382v1, 2017. 9, 20

MdPT19. Claudio Murolo, Andrew du Plessis, and David J. A. Trotman. Whitney cellulation of whitney stratified sets and goresky?s homology conjecture. hal preprint hal- hal02014662v1, 2019. 15

Mil94. Chris Miller. Exponentiation is hard to avoid. Proc. Amer. Math. Soc., 122(1):257-259, 1994. 10,14

Mos85. Tadeusz Mostowski. Lipschitz equisingularity. Dissertationes Math. (Rozprawy Mat.), 243:46, 1985. 7, 9

Nak00. Isao Nakai. Elementary topology of stratified mappings. In Singularities-Sapporo 1998, volume 29 of Adv. Stud. Pure Math., pages 221-243. Kinokuniya, Tokyo, 2000. 19

NAT81. Vicente Navarro Aznar and David J. A. Trotman. Whitney regularity and generic wings. Ann. Inst. Fourier (Grenoble), 31(2):v, 87-111, 1981. 13

NP12. Walter D. Neumann and Anne Pichon. Lipschitz geometry of complex surfaces: analytic invariants and equisingularity. arXiv preprint arXiv:1211.4897, 2012. 12

NTT14. Nhan Nguyen, Saurabh Trivedi, and David Trotman. A geometric proof of the existence of definable Whitney stratifications. Illinois J. Math., 58(2):381-389, 2014. 4

NV16. Nhan Nguyen and Guillaume Valette. Lipschitz stratifications in o-minimal structures. Ann. Sci. Éc. Norm. Supér. (4), 49(2):399-421, 2016. 7, 17

NV18. Nhan Nguyen and Guillaume Valette. Whitney stratifications and the continuity of local Lipschitz-Killing curvatures. Ann. Inst. Fourier (Grenoble), 68(5):2253-2276, 2018. 13

OT02. Patrice Orro and David Trotman. Cône normal et régularités de Kuo-Verdier. Bull. Soc. Math. France, 130(1):71-85, 2002. 11

Par88a. Adam Parusiński. Lipschitz properties of semi-analytic sets. Ann. Inst. Fourier (Grenoble), 38(4):189-213, 1988. 7

Par88b. Adam Parusiński. Lipschitz stratification of real analytic sets. In Singularities (Warsaw, 1985), volume 20 of Banach Center Publ., pages 323-333. PWN, Warsaw, 1988. 7

Par94. Adam Parusiński. Lipschitz stratification of subanalytic sets. Ann. Sci. École Norm. Sup. (4), 27(6):661-696, 1994. 7, 9

Par99. Adam Parusiński. Topological triviality of $\mu$-constant deformations of type $f(x)+\operatorname{tg}(x)$. Bull. London Math. Soc., 31(6):686-692, 1999. 19

PP17. Adam Parusiński and Laurenţiu Păunescu. Arc-wise analytic stratification, Whitney fibering conjecture and Zariski equisingularity. Adv. Math., 309:254-305, 2017. 9, 20

Paw85. Wiesław Pawłucki. Quasiregular boundary and Stokes's formula for a subanalytic leaf. In Seminar on deformations (Eódź/Warsaw, 1982/84), volume 1165 of Lecture Notes in Math., pages 235-252. Springer, Berlin, 1985. 14

RT97. Jean-Jacques Risler and David Trotman. Bi-Lipschitz invariance of the multiplicity. Bull. London Math. Soc., 29(2):200-204, 1997. 12

Sab83. Claude Sabbah Morphismes sans éclatements et cycles évanescents. in Analysis and topology on singular spaces II, III, Astérisque, Soc. Math. France, 101-102:286-319, 1983. 18

Shi97. Masahiro Shiota. Geometry of subanalytic and semialgebraic sets, volume 150 of Progress in Mathematics. Birkhäuser Boston, Inc., Boston, MA, 1997. 9 
Shi00. Masahiro Shiota. Thom's conjecture on triangulations of maps. Topology, 39(2):383399, 2000. 18

Shi05. Masahiro Shiota. Whitney triangulations of semialgebraic sets. Ann. Polon. Math., 87:237-246, 2005. 16

Shi10. Masahiro Shiota. Triangulations of non-proper semialgebraic Thom maps. In The Japanese-Australian Workshop on Real and Complex Singularities-JARCS III, volume 43 of Proc. Centre Math. Appl. Austral. Nat. Univ., pages 127-140. Austral. Nat. Univ., Canberra, 2010. 18

Tei73. Bernard Teissier. Cycles évanescents, sections planes et conditions de Whitney. In Singularités à Cargèse (Rencontre Singularités Géom. Anal., Inst. Études Sci., Cargèse, 1972), pages 285-362. Astérisque, Nos. 7 et 8. 1973. 19

Tei82. Bernard Teissier. Variétés polaires. II. Multiplicités polaires, sections planes, et conditions de Whitney. In Algebraic geometry (La Rábida, 1981), volume 961 of Lecture Notes in Math., pages 314-491. Springer, Berlin, 1982. 6, 13, 14

Tei89. Bernard Teissier Sur la triangulation des morphismes sous-analytiques. Publ. Math. de l'IHES, 70:169-198, 1989. 18

Tho69. René Thom. Ensembles et morphismes stratifiés. Bull. Amer. Math. Soc., 75:240-284, 1969. 4, 8, 18, 19

Tho65. René Thom. Propriétés différentielles locales des ensembles analytiques (d'après H. Whitney). In Séminaire Bourbaki, Vol. 9, pages Exp. No. 281, 69-80. Soc. Math. France, Paris, 1995. 4

Tri13. Saurabh Trivedi. Stratified transversality of holomorphic maps. Internat. J. Math., 24(13):1350106, 12, 2013. 8

Tro77. David Trotman. Whitney stratifications: faults and detectors. Warwick University thesis. 1977. 6, 8

Tro79. David J. A. Trotman. Geometric versions of Whitney regularity for smooth stratifications. Ann. Sci. École Norm. Sup. (4), 12(4):453-463, 1979. 5

Tro79. David J. A. Trotman. Stability of transversality to a stratification implies Whitney (a)-regularity. Invent. Math., 50(3):273-277, 1978/79. 8

TT14. Saurabh Trivedi and David Trotman. Detecting Thom faults in stratified mappings. Kodai Math. J., 37(2):341-354, 2014. 18

TV17. David Trotman and Guillaume Valette. On the local geometry of definably stratified sets. In Ordered algebraic structures and related topics, volume 697 of Contemp. Math., pages 349-366. Amer. Math. Soc., Providence, RI, 2017. 14, 15

TW06. David Trotman and Leslie Wilson. ( $r)$ does not imply $(n)$ or $(n p f)$ for definable sets in non polynomially bounded o-minimal structures. In Singularity theory and its applications, volume 43 of Adv. Stud. Pure Math., pages 463-475. Math. Soc. Japan, Tokyo, 2006. 14

Val05a. Guillaume Valette. A bilipschitz version of Hardt's theorem. C. R. Math. Acad. Sci. Paris, 340(12):895-900, 2005. 16, 17

Val05b. Guillaume Valette. Lipschitz triangulations. Illinois J. Math., 49(3):953-979, 2005. 17

Val08. Guillaume Valette. Volume, Whitney conditions and Lelong number. Ann. Polon. Math., 93(1):1-16, 2008. 13, 14

vdDM96. Lou van den Dries and Chris Miller. Geometric categories and o-minimal structures. Duke Math. J., 84(2):497-540, 1996. 4

Ver76. Jean-Louis Verdier. Stratifications de Whitney et théorème de Bertini-Sard. Invent. Math., 36:295-312, 1976. 4, 6, 9

Wal75. C. T. C. Wall. Regular stratifications. In Dynamical systems-Warwick 1974 (Proc. Sympos. Appl. Topology and Dynamical Systems, Univ. Warwick, Coventry, 1973/1974; presented to E. C. Zeeman on his fiftieth birthday), pages 332-344. Lecture Notes in Math., Vol. 468, 1975. 4

Whi65a. Hassler Whitney. Tangents to an analytic variety. Ann. of Math. (2), 81:496-549, 1965. 3,4 
Whi65b. Hassler Whitney. Local properties of analytic varieties. In Differential and Combinatorial Topology (A Symposium in Honor of Marston Morse), pages 205-244. Princeton Univ. Press, Princeton, N. J., 1965. 3, 9

Zar71. Oscar Zariski. Some open questions in the theory of singularities. Bull. Amer. Math. Soc., 77:481-491, 1971. 12 